\title{
ESPECIAÇÃO QUÍMICA DE ARSÊNIO INORGÂNICO NO ESTUÁRIO DA LAGUNA DOS PATOS (RS, BRASIL)
}

\author{
Josiane dos Santos Farias e Márcio Raimundo Milani* \\ Escola de Química e Alimentos, Universidade Federal do Rio Grande, CP 475, 96201-900 Rio Grande - RS, Brasil \\ Luis Felipe Hax Niencheski e Mariele Lopes de Paiva \\ Instituto de Oceanografia, Universidade Federal do Rio Grande, CP 474, 96201-900 Rio Grande - RS, Brasil
}

Recebido em 1/11/11; aceito em 6/3/12; publicado na web em 25/6/12

\begin{abstract}
CHEMICAL SPECIATION OF INORGANIC ARSENIC IN LAGUNA DOS PATOS ESTUARY (RS, BRAZIL). This study optimized and validated a method to perform chemical speciation of inorganic arsenic in water samples collected under the Monitoring Program of the Port of Rio Grande-RS in July and October 2010 from the Laguna dos Patos Estuary (RS, Brazil). The flow injection hydride generation atomic absorption spectrometry technique was employed, allowing quantification of $\mathrm{As}^{3+}$ and $\mathrm{As}^{5+}$ present in estuarine water samples. Data interpretation for results generated using the improved method for analyzing water samples collected from Laguna dos Patos Estuary was done by main components analysis.
\end{abstract}

Keywords: arsenic; speciation; flow injection hydride generation atomic absorption spectrometry.

\section{INTRODUÇÃO}

Os metais são contaminantes ambientais estáveis e persistentes, pois não podem ser degradados e nem destruídos. ${ }^{1} \mathrm{Na}$ natureza, os metais que ocorrem em baixas concentrações, variando entre 1 e $10^{-10}$ $\mathrm{mg} \mathrm{dm}{ }^{-3}$ são chamados de metais traço. ${ }^{2}$ Porém, a mineração no século $\mathrm{XX}$ aumentou consideravelmente a concentração destes elementos no meio ambiente aquático. Cabe salientar que não somente a mineração, as fontes antrópicas, como efluentes domésticos e industriais, o uso de pesticidas são responsáveis pela introdução dos metais nos ambientes aquáticos, mas também, as fontes naturais, como o intemperismo ou fenômeno wash-out de solos e rochas, que resultam na introdução desses elementos nos corpos hídricos. ${ }^{1}$

A contaminação das águas por metais traço vem chamando a atenção de pesquisadores, pois estes poluentes representam um risco em potencial à biota, devido ao seu caráter acumulativo. ${ }^{3}$

No meio ambiente, a mobilidade e a toxicidade do metal depende do estado de oxidação e da estrutura química da espécie metálica. ${ }^{4}$ Em particular, o estado de oxidação do arsênio desempenha um papel importante em seu comportamento ambiental e na sua toxicidade. ${ }^{5,6}$ $\mathrm{O}$ arsênio pode ocorrer na natureza em quatro estados de oxidação: $\mathrm{As}^{5+}, \mathrm{As}^{3+}, \mathrm{As}^{0}$ e $\mathrm{As}^{3-} \cdot{ }^{3,4,7}$ Pode ser encontrado na atmosfera, na água, em solos, sedimentos e organismos, ocorrendo naturalmente na crosta terrestre.

Segundo Borba et al., ${ }^{8}$ a toxicidade dos compostos de arsênio pode ser resumida na seguinte ordem: compostos de $\mathrm{As}^{3+}$ inorgânico $>$ compostos de $\mathrm{As}^{5+}$ inorgânico $>$ compostos de $\mathrm{As}^{3+}$ orgânico > compostos de $\mathrm{As}^{5+}$ orgânico. Os compostos de arsênio inorgânico são 100 vezes mais tóxicos do que as espécies químicas parcialmente metiladas (íon monometilarsônico (MMA) e íon dimetilarsínico (DMA)). ${ }^{9}$ Mesmo entre as espécies inorgânicas a toxicidade não é igual; por exemplo, a forma de arsênio trivalente (arsenito) é 60 vezes mais tóxica do que a forma oxidada pentavalente (arsenato)..$^{8,10,11}$

No meio ambiente, a contaminação por arsênio afeta diretamente as populações humanas. Esse elemento, uma vez presente na cadeia alimentar irá desencadear vários problemas potencialmente perigosos à saúde humana, podendo levar ao óbito. ${ }^{12}$

*e-mail: marcmilafurg@gmail.com
O consumo de água contaminada com altos teores de arsênio tem sido a principal causa de contaminação humana por esse elemento. Os casos mais graves de intoxicação por arsênio aconteceram em Bangladesh, Bengala Ocidental e também no México, Chile e Argentina, porque pessoas consumiram água subterrânea, extraída de aquíferos em formações geológicas arseníferas. ${ }^{13}$

A exposição crônica ao arsênio pode causar graves problemas metabólicos aos seres humanos, tais como, hiperqueratose, câncer de pele, câncer de fígado, câncer pulmonar, câncer de bexiga, câncer de rins, distúrbios do sistema nervoso, aumento da frequência de abortos espontâneos e outras doenças graves. ${ }^{4,12-15}$

Em função da sua toxicidade e possíveis impactos ambientais, continuam a ser desenvolvidas técnicas analíticas que permitam a detecção de arsênio em níveis traço. A espectrometria de absorção atômica e espectrometria de emissão óptica com plasma acoplado indutivamente são as técnicas mais utilizadas para a detecção de arsênio. Particularmente, a espectrometria de absorção atômica com atomização eletrotérmica (ETAAS) tem sido usada para determinar as concentrações de arsênio total em diferentes matrizes, por causa da sua sensibilidade e exatidão. ${ }^{10}$ No entanto, para análise de água do mar ou de águas não contaminadas por arsênio, esta técnica tem uso restrito, pois a concentração de arsênio encontrada nessas águas está abaixo do limite de detecção. ${ }^{10}$ A determinação somente é possível após uma etapa de pré-concentração e separação do analito, o que torna difícil sua utilização em análises de rotina.

Para a determinação da concentração de arsênio na faixa de alguns $\mu \mathrm{g} \mathrm{L}{ }^{-1}$, técnicas automatizadas como, por exemplo, a análise por injeção por fluxo (FIA) tem atraído atenção por sua alta precisão, elevada velocidade analítica e possibilidade de inclusão de pré-tratamentos e pré-concentração do analito em linha. Os métodos empregando a técnica de injeção em fluxo são os que asseguram uso de instrumentação mais simples e de baixo custo, além de permitir a determinação rápida de diversos elementos traço em linha. ${ }^{10}$

A análise por injeção em fluxo combinada com a espectrometria de absorção atômica com geração de hidretos (FI-HG-AAS) como sistema de detecção para arsênio é um acoplamento atrativo, por causa da sua simplicidade e alta sensibilidade. Quando comparado com o procedimento convencional, a FI-HG AAS apresenta os seguintes benefícios: sem perda do analito, devido à reação rápida e completa entre o metal e o hidreto gerado in situ (agente redutor); 
máxima eficiência de transporte do hidreto do metal formado, pois este se apresenta na forma gasosa; facilidade de separação do analito da matriz, eliminando problemas que podem surgir a partir das interferências espectrais; possibilidade de especiação química, para estados de oxidação com maior cinética de reação; aumento do limite de detecção, tipicamente de 5 a 30 vezes, comparado ao método convencional. ${ }^{3}$

A legislação brasileira estabelece valores máximos permitidos apenas para a concentração total de arsênio (incluindo o arsênio na fração dissolvida e na fração particulada, sem filtração da amostra). A Resolução no 357, de 17/3/2005, do Conselho Nacional do Meio Ambiente (CONAMA), que classifica as águas superficiais, estabelece limites máximos para a concentração de arsênio total que variam de 0,01 a $0,14 \mathrm{mg} \mathrm{L}^{-1}$, de acordo com a natureza e finalidade de uso da água. O Ministério da Saúde estabeleceu pela Portaria $n^{\circ} 518$, de 25/3/2004, que a concentração de arsênio total presente na água para consumo humano deve ser inferior a $0,01 \mathrm{mg} \mathrm{L}^{-1}$. Entretanto, a concentração total de arsênio não discrimina qual a concentração

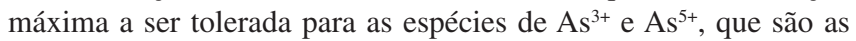
espécies mais tóxicas de arsênio.

Esse trabalho apresenta dados sobre a especiação química de arsênio inorgânico em amostras de água coletadas no estuário da Laguna dos Patos (RS, Brasil) na área de influência portuária do Porto de Rio Grande. As atividades portuárias, principalmente as operações de dragagem, podem contribuir significativamente com o aumento da concentração de arsênio inorgânico e, consequentemente, determinar a qualidade de suas águas. Sendo assim, foi proposta a otimização e adaptação de um método quantitativo, simples e de baixo custo baseado em trabalhos encontrados na literatura ${ }^{11,16-18}$ para a realização da especiação química de arsênio inorgânico.

Além disso, foi usada análise de fatores com análise dos componentes principais como ferramenta estatística para avaliar o comportamento das diferentes espécies de arsênio inorgânico com relação à concentração dos nutrientes inorgânicos dissolvidos $\left(\mathrm{NO}_{3}^{-}, \mathrm{NO}_{2}^{-}\right.$, $\mathrm{NH}_{4}{ }^{+}, \mathrm{PO}_{4}^{3-}, \mathrm{P}_{\text {total }}$ e $\mathrm{SiO}_{4}^{4-}$ ) e aos parâmetros químicos ( $\mathrm{pH}$, saturação de oxigênio, material em suspensão e condutividade) medidos em amostras na superfície e fundo da coluna d'água durante o inverno e a primavera de 2010 .

\section{PARTE EXPERIMENTAL}

\section{Equipamentos}

As concentrações de $\mathrm{As}^{3+}$ e $\mathrm{As}^{5+}$ foram determinadas pela técnica FIA-HG AAS, usando espectrômetro de absorção atômica AAnalyst600 (PerkinElmer) acoplado ao sistema FIAS-100 (PerkinElmer)

As concentrações de nutrientes nas amostras de água estuarina foram determinadas utilizando um espectrofotômetro (marca GBC UV/VIS, modelo 911A). Os parâmetros químicos foram medidos utilizando um salinômetro (marca YSI, modelo 30/10 FT), pHmetro (marca Mettler Toledo, modelo MP 120), oxímetro (marca Metter Toledo, modelo 128) e condutivímetro (marca Mettler Toledo, modelo MP 120).

\section{Limpeza do material}

A vidraria e os frascos de polietileno foram imersos em ácido nítrico $20 \%$ (v/v), durante 3 dias e, em seguida, por 1 dia em água ultrapura. Antes da imersão no ácido, todo o material foi enxaguado com água da torneira e 5 vezes com água destilada. O material foi seco em capela de fluxo laminar e embalado individualmente em sacos de polietileno. . $^{19,20}$

\section{Reagentes e soluções}

Os padrões e reagentes utilizados durante a análise foram de grau analítico ou superior e todas as soluções aquosas foram preparadas com água ultrapura obtida a partir de osmose reversa (Milli-RO, Millipore $^{\circledR}$ ) seguida por troca iônica (Milli-Q, Millipore ${ }^{\circledR}$ ).

Foi preparada solução estoque $100 \mathrm{mg} \mathrm{L}^{-1} \mathrm{de} \mathrm{As}^{3+}$ dissolvendo-se 0,0660 g de $\mathrm{As}_{2} \mathrm{O}_{3}$ p.a (Sigma-Aldrich, Missouri, EUA) em $20 \mathrm{~mL}$ de solução $1 \mathrm{~mol} \mathrm{~L}^{-1}$ de $\mathrm{NaOH}$, neutralizada com $20 \mathrm{~mL}$ de solução 2 mol L ${ }^{-1}$ de $\mathrm{HCl}$ e diluída até $1000 \mathrm{~mL}$ de solução a $0,6 \mathrm{~mol} \mathrm{~L}^{-1} \mathrm{HCl}^{11}$ A partir da solução estoque foi preparada a solução de trabalho de 5 $\mathrm{mg} \mathrm{L} \mathrm{L}^{-1} \mathrm{de} \mathrm{As}^{3+}$, por diluição com água ultrapura.

A solução de trabalho de $500 \mu \mathrm{g} \mathrm{L}^{-1}$ de $\mathrm{As}^{5+}$ foi preparada por diluição de solução estoque $1000 \mathrm{mg} \mathrm{L}^{-1}$, usada em espectrometria de absorção atômica (Spex Certiprep, New Jersey, EUA), com água ultrapura.

A solução tampão de citrato a $0,4 \mathrm{~mol} \mathrm{~L}^{-1}(\mathrm{pH}$ 6) foi preparada dissolvendo-se 11,764 g de citrato tri-sódio deca-hidratado em 80 $\mathrm{mL}$ de água ultrapura. Após a completa solubilização do sal, o pH da solução foi ajustado para 6 pela adição lenta de solução de ácido cítrico $1 \mathrm{~mol} \mathrm{~L}^{-1}$.

A solução pré-redutora $\left(5 \% \text { de } \mathrm{KI} \text { e } 5 \% \text { de } \mathrm{C}_{6} \mathrm{H}_{8} \mathrm{O}_{6}\right)^{11}$ e a solução redutora $\left(\mathrm{NaBH}_{4} 0,3 \%\right.$ em $\left.\mathrm{NaOH} 0,05 \%\right)$ foram preparadas diariamente.

\section{Amostras de água estuarina}

As amostras para a especiação de arsênio inorgânico foram coletadas ao longo da coluna d'água (superfície e fundo) em 11 estações de coleta distribuídas na Laguna dos Patos durante o inverno e a primavera de 2010, conforme a Figura 1S (material suplementar).

As amostras de água foram coletadas com auxílio de garrafa de Niskin na proa da embarcação. Uma fração da amostra foi filtrada através de membrana de acetato de celulose $0,45 \mu \mathrm{m}$ e transferida para frasco de polietileno de $500 \mathrm{~mL}$ e destinada à determinação da concentração dos nutrientes inorgânicos dissolvidos $\left(\mathrm{NO}_{3}^{-}, \mathrm{NO}_{2}^{-}\right.$, $\mathrm{NH}_{4}^{+}, \mathrm{PO}_{4}^{3-}, \mathrm{P}_{\text {total }}$ e $\mathrm{SiO}_{4}{ }^{4-}$ ) pelo método colorimétrico descrito por Baumgarten et al.. ${ }^{21}$

Uma segunda fração da mesma amostra que foi destinada à análise de arsênio inorgânico; imediatamente após a coleta, foi transferida para frascos de polietileno de $100 \mathrm{~mL}$ devidamente limpos e acondicionados em caixas de isopor, mantidas refrigeradas até a chegada ao laboratório.

No laboratório, as alíquotas para determinação de arsênio inorgânico foram filtradas através de membranas de acetato de celulose $0,20 \mu \mathrm{m}$ e mantidas resfriadas até a realização das análises.

\section{Procedimento para determinação das espécies inorgânicas de arsênio}

O método para quantificação das espécies de $\mathrm{As}^{3+}$ e $\mathrm{As}^{5+}$ foi adaptado e otimizado a partir de trabalhos já publicados. Foram investigadas a influência das concentrações das soluções carregadora e redutora $\mathrm{HCl}$ e $\mathrm{NaBH}_{4}$, respectivamente; o pH da solução tampão de citrato a $0,4 \mathrm{~mol} \mathrm{~L}^{-1}$, o tempo de reação e o volume de $\mathrm{HCl}$ concentrado usado na pré-redução de $\mathrm{As}^{5+}$. No sistema de injeção em fluxo sempre foram utilizadas as vazões indicadas pelo fabricante: $10 \mathrm{~mL} \mathrm{~min}{ }^{-1}$ para $\mathrm{HCl}, 6 \mathrm{~mL} \mathrm{~min}^{-1}$ para $\mathrm{NaBH}_{4}, 500 \mu \mathrm{L}$ de volume da amostra e $120 \mathrm{rpm}$ de velocidade da bomba peristáltica.

A determinação de $\mathrm{As}^{3+}$ consiste na redução das espécies de $\mathrm{As}^{3+}$ à arsina na presença de $\mathrm{NaBH}_{4} 0,3 \%(\mathrm{~m} / \mathrm{V}), \mathrm{HCl} 3 \mathrm{~mol} \mathrm{~L}^{-1}$, solução tampão de citrato a $0,4 \mathrm{~mol} \mathrm{~L}^{-1}(\mathrm{pH}=6$; concentração final de $32 \mathrm{mmol}$ $\left.\mathrm{L}^{-1}\right) . \mathrm{O} \mathrm{As}^{5+}$ foi mensurado por determinação indireta da concentração 
de arsênio inorgânico total, após a redução das espécies de arsênio no estado de oxidação $\mathrm{V}$ para o estado de oxidação III com adição de $10 \%$ do volume final da solução de $\mathrm{C}_{6} \mathrm{H}_{8} \mathrm{O}_{6} / \mathrm{KI}$ (concentração final $0,5 \%$ de $\mathrm{C}_{6} \mathrm{H}_{8} \mathrm{O}_{6}$ e $0,5 \%$ de $\mathrm{KI}$ ), de $6 \%$ do volume final de $\mathrm{HCl}$ p.a. (concentração final 7,1 $\mathrm{mmol} \mathrm{L}^{-1}$ ) e tempo de reação de $60 \mathrm{~min}$, utilizando as mesmas concentrações de $\mathrm{HCl}$ e $\mathrm{NaBH}_{4}$ como descrito anteriormente para determinação de $\mathrm{As}^{3+}$. A concentração de $\mathrm{As}^{5+}$ foi obtida pela subtração da concentração de arsênio inorgânico total pela concentração de $\mathrm{As}^{3+}$.

\section{RESULTADOS E DISCUSSÃO}

\section{Validação}

Após a otimização do método analítico proposto para determinação das diferentes espécies de arsênio inorgânico $\left(\mathrm{As}^{3+} \mathrm{e} \mathrm{As}^{5+}\right)$, a validação do método incluindo linearidade, limites de detecção e de quantificação, precisão e exatidão foi investigada em amostras de água salgada (salinidade 35) coletada próximo aos Molhes da Barra (Rio Grande, RS) e de água doce (salinidade 0) coletada de um lago próximo à FURG.

Para verificar a linearidade foram construídas duas curvas de calibração no intervalo de 1 a $30 \mu \mathrm{g} \mathrm{L}^{-1}$. A curva de calibração para $\mathrm{As}^{3+}$ apresentou coeficiente de correlação (r) de 0,9999 e equação da reta $y=0,0151[\mathrm{As}]+0,0052$. Para $\mathrm{As}^{5+}$ foi obtida uma curva de calibração com coeficiente de correlação (r) de 0,9999 e equação da reta $\mathrm{y}=0,0053[\mathrm{As}]+0,0072$.

A precisão foi expressa em termos de repetitividade, através do cálculo da estimativa do desvio-padrão relativo (RSD). As amostras foram fortificadas com 1,8 e $30 \mu \mathrm{g} \mathrm{L}^{-1}$ de $\mathrm{As}^{3+} \mathrm{e} \mathrm{As}^{5+}(n=3)$. Os valores de RSD encontrados para $\mathrm{As}^{3+}$ nas amostras de água salgada foram 7,$8 ; 2,2$ e $2,7 \%$. Nas amostras de água doce os valores foram 11,7; 6,3 e 1,3\%. Para $\mathrm{As}^{5+}$ os valores de RSD encontrados na amostra de água salgada foram 5,8; 2,6 e 2,6\%. Para água doce foram encontrados valores de 13,$1 ; 3,3$ e $1,9 \%$.

O LOD e o LOQ foram estimados considerando os critérios de 3 desvios-padrões (3s) e de 10 desvios-padrões (10s), respectivamente. Para os cálculos dos respectivos LOD e LOQ, tanto para o método de determinação de $\mathrm{As}^{3+}$ quanto para $\mathrm{As}^{5+}$, foram feitas 6 repetições de uma solução de $\mathrm{As}^{3+} \mathrm{e} \mathrm{As}^{5+} 1 \mu \mathrm{g} \mathrm{L}^{-1}$. Os limites de detecção para $\mathrm{As}^{3+} \mathrm{e} \mathrm{As}^{5+}$ foram 0,192 e $0,313 \mu \mathrm{g} \mathrm{L}^{-1}$, respectivamente. Os limites de quantificação foram 0,575 para $\mathrm{As}^{3+}$ e $0,939 \mu \mathrm{g} \mathrm{L} \mathrm{L}^{-1}$ para As ${ }^{5+}$.

A exatidão foi avaliada pelo método ou teste de adição e recuperação realizados em amostras de águas doce e salgada. Nas duas matrizes de água foram realizadas fortificações de 1,8 e $30 \mu \mathrm{g} \mathrm{L}^{-1} \mathrm{com}$

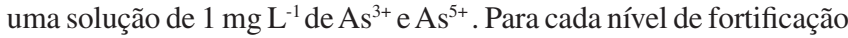
foram realizadas três repetições. Os resultados para as recuperações de $\mathrm{As}^{3+}$ nas amostras de água doce fortificadas com 1,8 e $30 \mu \mathrm{g} \mathrm{L}^{-1}$ foram 99,$1 ; 105,0$ e 94,8\%. As recuperações de $\mathrm{As}^{3+}$ nas amostras de água salgada fortificadas com os mesmos níveis de concentração foram 87,$5 ; 96,6$ e 91,4\%, respectivamente. Os valores obtidos para as recuperações de $\mathrm{As}^{5+}$ nas amostras de água doce fortificadas com 1,8 e $30 \mu \mathrm{g} \mathrm{L}^{-1}$ foram 79,1; 79,9 e 97,9\%. Para as amostras de água salgada fortificadas com os mesmos níveis de concentração de $\mathrm{As}^{5+}$ foram obtidas recuperações de 114,6; 100,4 e 86,7\%, respectivamente.

\section{Especiação de arsênio inorgânico na Laguna dos Patos}

A concentração de $\mathrm{As}^{3+}$ na primavera foi superior à concentração de $\mathrm{As}^{3+}$ no inverno, tanto na superfície quanto no fundo da coluna d'água na Laguna dos Patos. Já a concentração de $\mathrm{As}^{5+}$ no inverno foi maior que a concentração de $\mathrm{As}^{5+}$ encontrada na primavera, nos respectivos níveis da coluna d'água. Os resultados das concentrações das diferentes espécies de arsênio na superfície e no fundo da coluna d'água no inverno e na primavera na Laguna dos Patos são mostrados nas Tabelas $1 \mathrm{~S}$ e $2 \mathrm{~S}$ (material suplementar), respectivamente.

No entanto, não se pode afirmar se a concentração encontrada está abaixo ou acima do limite estipulado pela legislação, pois a legislação brasileira contempla apenas a concentração de arsênio total e algumas legislações estrangeiras estabelecem no máximo a concentração de alguns metais na fração dissolvida, não apresentando nenhuma informação quanto à especiação química, sendo fundamental o seu conhecimento, principalmente em se tratando de metais pesados, que representam um risco em potencial ao ambiente aquático.

\section{Determinação dos nutrientes inorgânicos dissolvidos e dos parâmetros químicos}

As concentrações dos nutrientes inorgânicos dissolvidos nas amostras de água estuarina foram determinadas conforme descrito anteriormente. ${ }^{21}$ Os parâmetros químicos das amostras foram medidos in situ. Os resultados da concentração dos nutrientes inorgânicos dissolvidos e dos parâmetros químicos analisados são mostrados nas Tabelas 3S a 6S (material suplementar).

\section{Tratamento estatístico}

Os dados monitorados, nos dois níveis da coluna d'água (superfície e fundo) e nos período de inverno e primavera, foram tratados por análise fatorial com análise de componentes principais.

Primeiramente, os dados originais foram padronizados de modo que a média ficasse igual a 0 e o desvio padrão igual a 1 . Após, os fatores foram extraídos através da análise de componentes principais (ACP) utilizando o programa computacional Statistica 7.0 ${ }^{\circledR}$. A escolha do número de fatores foi feita pelo critério de Kaiser. ${ }^{22}$ Além disso, foi feita a rotação dos fatores pelo método Varimax Normalizada. ${ }^{23}$

As Tabelas 7S e 8S (material suplementar) mostram os 5 primeiros fatores importantes extraídos e os respectivos percentuais de variação explicada para a superfície e o fundo da coluna d'água no período de inverno.

De acordo com a Tabela 7S, material suplementar, os 5 primeiros fatores explicaram $83,9 \%$ da variância; segundo a Tabela $8 \mathrm{~S}$, os 5 fatores escolhidos apresentaram um percentual de variância de $85,3 \%$. Esses fatores extraídos pela análise estatística possuem um percentual de explicação adequado para interpretação dos resultados gerados a partir dos dados originais. Apesar dos 5 primeiros fatores apresentarem um bom percentual de explicação segundo o critério de Kaiser, somente os 4 primeiros foram usados no tratamento estatístico dos dados da superfície e do fundo da coluna d'água no período de inverno, pois o quinto fator apresentou uma baixa contribuição na explicação dos resultados (apenas 9,8 e 13,2\% de explicação da variância encontrada, respectivamente).

Cabe salientar que apenas os dois primeiros fatores foram interpretados neste estudo, pois foram os fatores que apresentaram um maior percentual de explicação da variabilidade dos dados. Esses fatores agruparam as variáveis que apresentaram uma maior variação em comum e, a partir deles, foi possível a interpretação dos dados. Além disso, foram os únicos fatores que contribuíram com a explicação do modelo construído pela análise, pois apresentaram autovalores altos. Ou seja, esses dois fatores auxiliaram na compreensão dos fenômenos que ocorreram na Laguna dos Patos nos períodos de inverno e de primavera de 2010 .

As Tabelas 9S e 10S (material suplementar) apresentam os resultados dos 4 primeiros fatores extraídos para a superfície e o fundo da coluna d'água no período da primavera, os quais são os mais importantes para a interpretação dos dados. Esses fatores juntos 
representaram 80,1 e 79,7\% de explicação da variância encontrada, respectivamente.

\section{Período de inverno (superfície)}

O resultado da rotação dos fatores pelo método Varimax Normalizada com as cargas fatoriais das 12 variáveis para cada fator é mostrado na Tabela 1.

As variáveis que possuem uma carga fatorial de 0,5 ou mais, mostradas na Tabela 1, estão em negrito, pois essas cargas fatoriais indicam o grau de correlação entre a variável original e os fatores.

$\mathrm{O}$ fator 1 apresentou o maior autovalor $(3,33$; Tabela $7 \mathrm{~S}$, material suplementar) e teve uma explicação de $27,82 \%$ da variabilidade total dos dados. O fator 1 foi representado pela condutividade $(0,91$; $\mathrm{p}>0,05)$. A partir desta variável foi possível interpretar o fator $1, o$ qual pode ser representativo da variação da salinidade, uma vez que a condutividade mede a presença de íons majoritários, sobretudo os íons $\mathrm{Cl}^{-}$.

O fator 2 é responsável pela explicação de 20,19\% da variabilidade total dos dados. As variáveis que compartilharam esse fator foram $\mathrm{NH}_{4}{ }^{+}(0,58 ; \mathrm{p}>0,05), \mathrm{PO}_{4}{ }^{3-}(-0,82 ; \mathrm{p}>0,05)$ e $\mathrm{SiO}_{4}{ }^{4-}(0,80 ; \mathrm{p}>0,05)$. Sendo assim, o fator 2 pode ser representativo da ressuspensão dos sedimentos. Os aportes de $\mathrm{NH}_{4}{ }^{+}$e $\mathrm{PO}_{4}{ }^{3-}$ podem estar relacionados com a ressuspensão dos sedimentos, por causa da forte hidrodinâmica apresentada no período amostral, liberando $\mathrm{NH}_{4}{ }^{+}$presente na água intersticial da coluna sedimentar para a coluna d'água e, também, contribuindo com a variação da concentração de $\mathrm{PO}_{4}{ }^{3-}$ na superfície e $\mathrm{SiO}_{4}{ }^{4-}$ no processo de dissolução dos sedimentos silicosos abundantes nesta região.

\section{Período de inverno (fundo)}

Os resultados dos quatro primeiros fatores extraídos pelo método da ACP, utilizando o método de rotação Varimax Normalizada são apresentados na Tabela 2.

O fator 1 foi derivado do maior autovalor $(2,68$; Tabela $8 \mathrm{~S}$, material suplementar) e teve uma explicação de $22,36 \%$. As variáveis que mais contribuíram neste fator foram o $\mathrm{pH}(0,87 ; \mathrm{p}>0,05)$ e $\mathrm{NH}_{4}^{+}$ $(0,74 ; p>0,05)$. Dessa forma, o fator 1 também pode ser representativo da ressuspensão dos sedimentos, pois este fenômeno pode ser responsável pelo aporte de $\mathrm{NH}_{4}^{+}$para a Laguna dos Patos.

O fator 2 foi responsável pela explicação de $19,21 \%$ e foi representado apenas pela variável $\mathrm{As}^{3+}(-0,86 ; \mathrm{p}>0,05)$. Este fator pode ser representativo da falta de contaminação de $\mathrm{As}^{3+}$ no estuário.

\section{Período da primavera (superfície)}

Os resultados obtidos pela rotação Varimax Normalizada dos quatro primeiros fatores extraídos pela ACP são mostrados na Tabela 3 .

$\mathrm{O}$ fator 1 foi originado do maior autovalor $(4,03$; Tabela $9 \mathrm{~S}$, material suplementar) e explicou $33,58 \%$ da variabilidade dos dados. Este fator foi representado pelas variáveis MS (material em suspensão; 0,$88 ; \mathrm{p}>0,05), \mathrm{NH}_{4}^{+}(0,80 ; \mathrm{p}>0,05)$ e $\mathrm{As}^{3+}(0,56$; $\mathrm{p}>0,05)$ e, também, pela variável saturação de $\mathrm{O}_{2}(-0,68 ; \mathrm{p}>0,05)$.

Tabela 1. Principais fatores extraídos a partir dos dados originais da superfície da coluna d'água no período de inverno pelo método ACP

\begin{tabular}{lccccc}
\hline Variáveis & Fator 1 & Fator 2 & Fator 3 & Fator 4 & Fator 5 \\
\hline condutividade & $\mathbf{0 , 9 1 8 3 9 4}$ & 0,062848 & 0,083728 & 0,012414 & $-0,120863$ \\
$\mathrm{pH}$ & $-0,176242$ & 0,036049 & 0,228344 & 0,031525 & $-0,025453$ \\
saturação de $\mathrm{O}_{2}$ & 0,207299 & $-0,358258$ & 0,127160 & $\mathbf{- 0 , 6 6 4 4 0 8}$ & $-0,180719$ \\
$\mathrm{MS}$ & $-0,017606$ & 0,146513 & $-0,079586$ & $\mathbf{- 0 , 6 1 5 8 8 3}$ & $\mathbf{0 , 5 6 5 4 6 1}$ \\
$\mathrm{NH}_{4}^{+}$ & $-0,451474$ & $\mathbf{0 , 5 8 5 3 8 3}$ & $-0,004440$ & $-0,103568$ & 0,157172 \\
$\mathrm{PO}_{4}{ }^{3-}$ & $-0,036300$ & $\mathbf{- 0 , 8 2 4 3 5 0}$ & 0,440834 & 0,078512 & 0,121685 \\
$\mathrm{P}_{\text {total }}$ & 0,078847 & $-0,242771$ & 0,157617 & $\mathbf{0 , 9 4 1 9 6 8}$ & 0,126156 \\
$\mathrm{NO}_{2}^{-}$ & $-0,209137$ & 0,002961 & 0,194987 & 0,096755 & $-0,054211$ \\
$\mathrm{NO}_{3}^{-}$ & $-0,121875$ & 0,083599 & 0,035356 & 0,153524 & $\mathbf{0 , 9 7 3 7 7 8}$ \\
$\mathrm{SiO}_{4}{ }^{4-}$ & 0,115165 & $\mathbf{0 , 8 0 8 7 5 3}$ & 0,209053 & $-0,170515$ & 0,345845 \\
$\mathrm{As}^{3+}$ & 0,218829 & $-0,059126$ & $-0,035992$ & $-0,058103$ & 0,036029 \\
$\mathrm{As}^{5+}$ & 0,080369 & $-0,074622$ & $\mathbf{0 , 9 4 8 1 0 1}$ & 0,110299 & 0,014850 \\
\% da variância explicada & 27,82517 & 20,19193 & 14,28291 & 11,79455 & 9,77889 \\
\hline
\end{tabular}

Tabela 2. Principais fatores extraídos a partir dos dados originais do fundo da coluna d'água no período de inverno pelo método ACP

\begin{tabular}{lccccc}
\hline Variáveis & Fator 1 & Fator 2 & Fator 3 & Fator 4 & Fator 5 \\
\hline condutividade & 0,032616 & 0,160560 & $\mathbf{0 , 6 7 7 7 9 9}$ & $-0,398463$ & $-0,413828$ \\
$\mathrm{pH}$ & $\mathbf{0 , 8 7 1 0 7 4}$ & $-0,112527$ & $-0,044559$ & $-0,065814$ & $-0,073098$ \\
saturação de $\mathrm{O}_{2}$ & $-0,199473$ & 0,031354 & $\mathbf{- 0 , 7 3 9 6 9 7}$ & $-0,007172$ & $-0,319060$ \\
$\mathrm{MS}$ & 0,296875 & 0,192228 & 0,015786 & 0,001826 & $-0,262589$ \\
$\mathrm{NH}_{4}^{+}$ & $\mathbf{0 , 7 4 8 8 1 3}$ & 0,401202 & 0,078660 & 0,231812 & 0,330462 \\
$\mathrm{PO}_{4}{ }^{3-}$ & $-0,249522$ & 0,148564 & $-0,002501$ & $-0,090390$ & 0,189071 \\
$\mathrm{P}_{\text {total }}$ & 0,071635 & $-0,000401$ & 0,055959 & $-0,165582$ & $\mathbf{0 , 9 3 4 5 7 9}$ \\
$\mathrm{NO}_{2}^{-}$ & 0,002320 & 0,310216 & $-0,002344$ & 0,298716 & 0,082946 \\
$\mathrm{NO}_{3}^{-}$ & $-0,057368$ & 0,196981 & 0,055350 & $\mathbf{- 0 , 9 3 6 8 6 4}$ & 0,159764 \\
$\mathrm{SiO}_{4}{ }^{4-}$ & 0,069790 & 0,060656 & 0,160272 & 0,024585 & $-0,136683$ \\
$\mathrm{As}^{3+}$ & $-0,061196$ & $\mathbf{- 0 , 8 6 4 3 2 5}$ & 0,131682 & 0,298853 & 0,035569 \\
$\mathrm{As}^{5+}$ & $-0,109038$ & $-0,169535$ & $\mathbf{0 , 9 0 0 8 5 7}$ & 0,034798 & 0,035659 \\
$\%$ da variância explicada & 22,36624 & 19,21157 & 16,40073 & 14,10182 & 13,18865 \\
\hline
\end{tabular}


Tabela 3. Principais fatores extraídos a partir dos dados originais da superfície da coluna d'água no período da primavera pelo método ACP

\begin{tabular}{lcccc}
\hline Variáveis & Fator 1 & Fator 2 & Fator 3 & Fator 4 \\
\hline condutividade & $-0,008260$ & $\mathbf{0 , 9 1 8 8 2 1}$ & $-0,021312$ & 0,205578 \\
$\mathrm{pH}$ & $-0,129719$ & $\mathbf{0 , 8 9 7 0 1 8}$ & 0,292260 & $-0,213649$ \\
saturação de $\mathrm{O}_{2}$ & $\mathbf{- 0 , 6 8 0 3 9 2}$ & 0,260658 & 0,158790 & $-0,047683$ \\
$\mathrm{MS}^{-}$ & $\mathbf{0 , 8 8 7 7 6 4}$ & 0,012859 & 0,250059 & 0,081874 \\
$\mathrm{NH}_{4}{ }^{+}$ & $\mathbf{0 , 8 0 7 0 5 2}$ & $-0,018504$ & $-0,155455$ & $-0,013840$ \\
$\mathrm{PO}_{4}{ }^{3-}$ & 0,253810 & $-0,010888$ & 0,049990 & 0,126618 \\
$\mathrm{P}_{\text {total }}$ & 0,073595 & 0,190928 & $\mathbf{0 , 9 3 9 1 2 5}$ & $-0,099887$ \\
$\mathrm{NO}_{2}{ }^{-}$ & 0,378239 & $-0,076303$ & $-0,133032$ & 0,179911 \\
$\mathrm{NO}_{3}{ }^{-}$ & 0,161803 & 0,229766 & $-0,205689$ & 0,220197 \\
$\mathrm{SiO}_{4}{ }^{-}$ & 0,025969 & 0,000635 & $-0,106551$ & $\mathbf{0 , 9 2 3 4 7 9}$ \\
$\mathrm{As}^{3+}$ & $\mathbf{0 , 5 6 9 4 5 4}$ & $-0,325106$ & 0,307514 & $-0,261041$ \\
$\mathrm{As}^{5+}$ & 0,000792 & 0,293957 & 0,103176 & 0,215836 \\
$\%$ da variância & 33,58927 & 21,60718 & 15,97405 & 9,01330 \\
explicada & & & & \\
\hline
\end{tabular}

Sendo assim, o fator 1 pode ser representativo da ressuspensão dos sedimentos, pois as atividades portuárias, principalmente, aquelas do terminal de carga e descarga de grãos vegetais, por exemplo, podem liberar detritos de matéria orgânica que são depositados no sedimento para a coluna d'água contribuindo, assim, para o aumento da concentração de $\mathrm{NH}_{4}{ }^{+}$no ambiente em estudo. Além disso, existe a influência antrópica dos efluentes domésticos e industriais não tratados, que podem ser grandes fontes de $\mathrm{NH}_{4}{ }^{+}$e arsênio paras as águas em estudo.

O fator 2 foi responsável por $21,60 \%$ da explicação da variabilidade dos dados, sendo representado por apenas duas variáveis: $\mathrm{pH}(0,89 ; \mathrm{p}>0,05)$ e condutividade $(0,91 ; \mathrm{p}>0,05)$. Esse fator pode ser representativo da variação de salinidade no ambiente em estudo.

\section{Período da primavera (fundo)}

Os resultados dos 4 primeiros fatores extraídos pela ACP com rotação Varimax Normalizada estão na Tabela 4.

Tabela 4. Principais fatores extraídos a partir dos dados originais do fundo da coluna d'água no período da primavera pelo método ACP

\begin{tabular}{lcccc}
\hline Variáveis & Fator 1 & Fator 2 & Fator 3 & Fator 4 \\
\hline condutividade & 0,087300 & $\mathbf{0 , 9 1 9 1 6 9}$ & 0,030252 & $-0,044349$ \\
$\mathrm{pH}$ & $-0,125492$ & $\mathbf{0 , 9 8 7 6 7 7}$ & 0,037251 & 0,022146 \\
saturação de $\mathrm{O}_{2}$ & $-0,155550$ & $\mathbf{- 0 , 5 1 5 6 4 8}$ & $-0,469998$ & 0,253288 \\
$\mathrm{MS}^{+}$ & $\mathbf{0 , 9 6 6 6 4 9}$ & $-0,011799$ & $-0,101934$ & 0,169391 \\
$\mathrm{NH}_{4}{ }^{-}$ & 0,141935 & $-0,334197$ & 0,400483 & $-0,469586$ \\
$\mathrm{PO}_{4}{ }^{-}$ & 0,077225 & $-0,131660$ & 0,005493 & 0,133523 \\
$\mathrm{P}_{\text {total }}$ & 0,343106 & $-0,287858$ & 0,068642 & 0,336377 \\
$\mathrm{NO}_{2}{ }^{-}$ & 0,039299 & 0,024000 & 0,229125 & 0,040936 \\
$\mathrm{NO}_{3}^{-}$ & 0,215300 & $-0,041213$ & 0,105575 & $\mathbf{0 , 9 4 6 1 7 1}$ \\
$\mathrm{SiO}_{4}{ }^{-}$ & $-0,127704$ & 0,267388 & 0,122260 & $-0,118978$ \\
$\mathrm{As}^{3+}$ & $-0,170548$ & 0,066597 & $\mathbf{0 , 9 4 0 4 2 3}$ & 0,090485 \\
$\mathrm{As}^{5+}$ & $\mathbf{- 0 , 5 4 7 6 9 2}$ & 0,053045 & 0,202003 & $\mathbf{- 0 , 5 4 2 6 2 9}$ \\
$\%$ da variância & 35,28386 & 18,25808 & 16,97546 & 9,26098 \\
explicada $^{-}$ & & & & \\
\hline
\end{tabular}

O fator 1 foi responsável pela explicação de 35,28\% dos dados (maior porcentagem de explicação da variabilidade dos dados) e, além disso, foi originado do maior autovalor $(4,23$; Tabela $10 \mathrm{~S}$, material suplementar). Neste fator, as variáveis representativas foram o MS (material em suspensão; 0,$96 ; p>0,05)$ e $\mathrm{As}^{5+}(-0,54 ; \mathrm{p}>0,05)$. Dessa maneira, o fator 1 pode ser representativo do carreamento do material particulado pelo estuário da Laguna dos Patos.

$\mathrm{O}$ fator 2 foi representado pelas variáveis $\mathrm{pH}(0,98 ; \mathrm{p}>0,05)$, condutividade $(0,91 ; p>0,05)$ e saturação de $\mathrm{O}_{2}(-0,51 ; \mathrm{p}>0,05)$ explicando $18,25 \%$ da variabilidade total dos dados. Sendo assim, o fator 2 pode ser representativo da variação de salinidade.

\section{CONCLUSÃO}

Os dados tratados estatisticamente revelaram que a concentração de arsênio na superfície da coluna d'água na primavera na Laguna dos Patos está relacionada com a concentração de $\mathrm{NH}_{4}{ }^{+}$e, também, com a concentração de material em suspensão, pois apresentaram uma relação significativa entre si.

Os resultados mostram que não houve nenhuma alteração significativa das concentrações de $\mathrm{As}^{3+}$ e de $\mathrm{As}^{5+}$ na superfície e no fundo do estuário da Laguna dos Patos, pois as concentrações destas espécies permaneceram as mesmas.

Como mencionado anteriormente, a concentração de $\mathrm{As}^{3+}$ foi maior na primavera do que no inverno, e a concentração de $\mathrm{As}^{5+}$ foi maior no inverno do que na primavera nos dois níveis da coluna d'água, apresentando comportamentos distintos. Dessa maneira, os resultados indicam que pode estar ocorrendo algum tipo de atividade que esteja favorecendo o aumento da concentração de $\mathrm{As}^{3+}$ na primavera e, também, o aumento da concentração de $\mathrm{As}^{5+}$ no inverno, havendo a necessidade de estudos mais aprofundados para se conhecer a possível fonte desta elevação da concentração de ambas as espécies inorgânicas de arsênio.

Além disso, este estudo se torna relevante dada a importância da adequação da legislação brasileira em relação à especiação química inorgânica de arsênio para estabelecer valores não apenas para a fração total de arsênio, mas também, para $\mathrm{As}^{3+}$ e $\mathrm{As}^{5+}$, que são as espécies inorgânicas mais tóxicas para o meio ambiente.

\section{MATERIAL SUPLEMENTAR}

No material suplementar estão a Figura 1S apresentando o mapa com as 11 estações de coleta e as Tabelas 1S a 10S. Este material está disponível em http://quimicanova.sbq.org.br, na forma de arquivo PDF, com acesso livre.

\section{AGRADECIMENTOS}

J. S. Farias agradece ao CNPq pela bolsa concedida, à Superintendência do Porto de Rio Grande e ao laboratório de Hidroquímica.

\section{REFERÊNCIAS}

1. Silva, S. C.; Dissertação de Mestrado, Universidade de São Paulo, Brasil, 2008.

2. Niencheski, L. F. H.; Milani, I. C. B.; Milani, M. R. Em Poluição Marinha; Neto, J. A. B.; Wallner- Kersanach, M.; Patchineelam, S. M., orgs.; Interciência: Rio de Janeiro, 2008, cap. 7.

3. Assis, R. A.; Tese de Doutorado, Pontifícia Universidade Católica do Rio de Janeiro, Brasil, 2006.

4. Hung, D. Q.; Nekrassova, O.; Compton, R. G.; Talanta 2004, 64, 269.

5. Cabon, J. Y.; Cabon, N.; Anal. Chim. Acta 2000, 418, 19.

6. Coelho, N. M. M.; Cósmen da Silva, A.; Moraes da Silva, C.; Anal. Chim. Acta 2002, 460, 227.

7. Leermakers, M.; Baeyens, W.; De Gieter, M.; Smedts, B.; Meert, C.; De Bisschop, H. C.; Morabito, R.; Quevauviller, Ph.; Trends Anal. Chem. 2006, 25, 1 . 
8. Borba, R. P.; Coscione, A. R.; Figueiredo, B. R.; Zambello, F.; Quim. Nova 2009, 32, 970.

9. Szkoda, J.; Zmudzki, J.; Grzebalska, A.; Bull. Vet. Inst. Pulawy 2006, 50, 269.

10. Barra, C. M.; Santelli, R. E.; Abrão, J. J.; de la Guardia, M.; Quim. Nova 2000, 23, 58 .

11. Sigrist, M. E.; Beldoménico, H. R.; Spectrochim. Acta, Part B 2004, 59, 1041.

12. Pereira, S. F. P.; Oliveira, G. R. F.; Oliveira, J. S.; Silva, J. S.; Sousa Júnior, P. M.; Acta Amaz. 2009, 39, 953.

13. Figueiredo, B. R.; Borba, R. P.; Angélica, R. S. Em Geologia Médica no Brasil; Silva, C. R.; Figueiredo, B. R.; De Capitani, E. M.; Cunha, F. G., eds.; CPRM-Serviço geológico do Brasil: Rio de Janeiro, 2006, cap. 11.

14. Howard, A. G.; Arbab-Zavar, M. H.; Analyst 1980, 105, 744.

15. Mazumder, D. N.; Indian J. Med. Res. 2008, 128, 436.
16. Chanthai, S.; Suwamat, N.; Ruangviriyachai, C.; Danvirutai, P.; ASEAN Food J. 2007, 14, 181.

17. Quináia, S. P.; Rollemberg, M. C. E.; J. Braz. Chem. Soc. 1997, 8, 349.

18. Quináia, S. P.; Rollemberg, M. C. E.; J. Braz. Chem. Soc. 2001, 12, 37.

19. Borges, A. R.; Dissertação de Mestrado, Universidade Federal do Rio Grande, Brasil, 2009.

20. Milani, I. C. B.; Dissertação de Mestrado, Universidade Federal do Rio Grande, Brasil, 2004.

21. Baumgarten, M. G. Z.; Rocha, J. M. B.; Niencheski, L. F. H.; Manual de análises em Oceanografia Química, $1^{\mathrm{a}}$ ed., Editora da Furg: Rio Grande, 1996.

22. Bezerra, F. A. Em Análise Fatorial; Corrar, L. J.; Paulo, E.; Filho, J. M. D., coords.; Atlas: São Paulo, 2009, cap. 2.

23. Reis, E.; Estatística Multivariada, $2^{\mathrm{a}}$ ed., Edições Sílabo: Lisboa, 2001. 


\section{ESPECIAÇÃO QUÍMICA DE ARSÊNIO INORGÂNICO NO ESTUÁRIO DA LAGUNA DOS PATOS (RS, BRASIL)}

Josiane dos Santos Farias e Márcio Raimundo Milani*

Escola de Química e Alimentos, Universidade Federal do Rio Grande, CP 475, 96201-900 Rio Grande - RS, Brasil Luis Felipe Hax Niencheski e Mariele Lopes de Paiva

Instituto de Oceanografia, Universidade Federal do Rio Grande, CP 474, 96201-900 Rio Grande - RS, Brasil

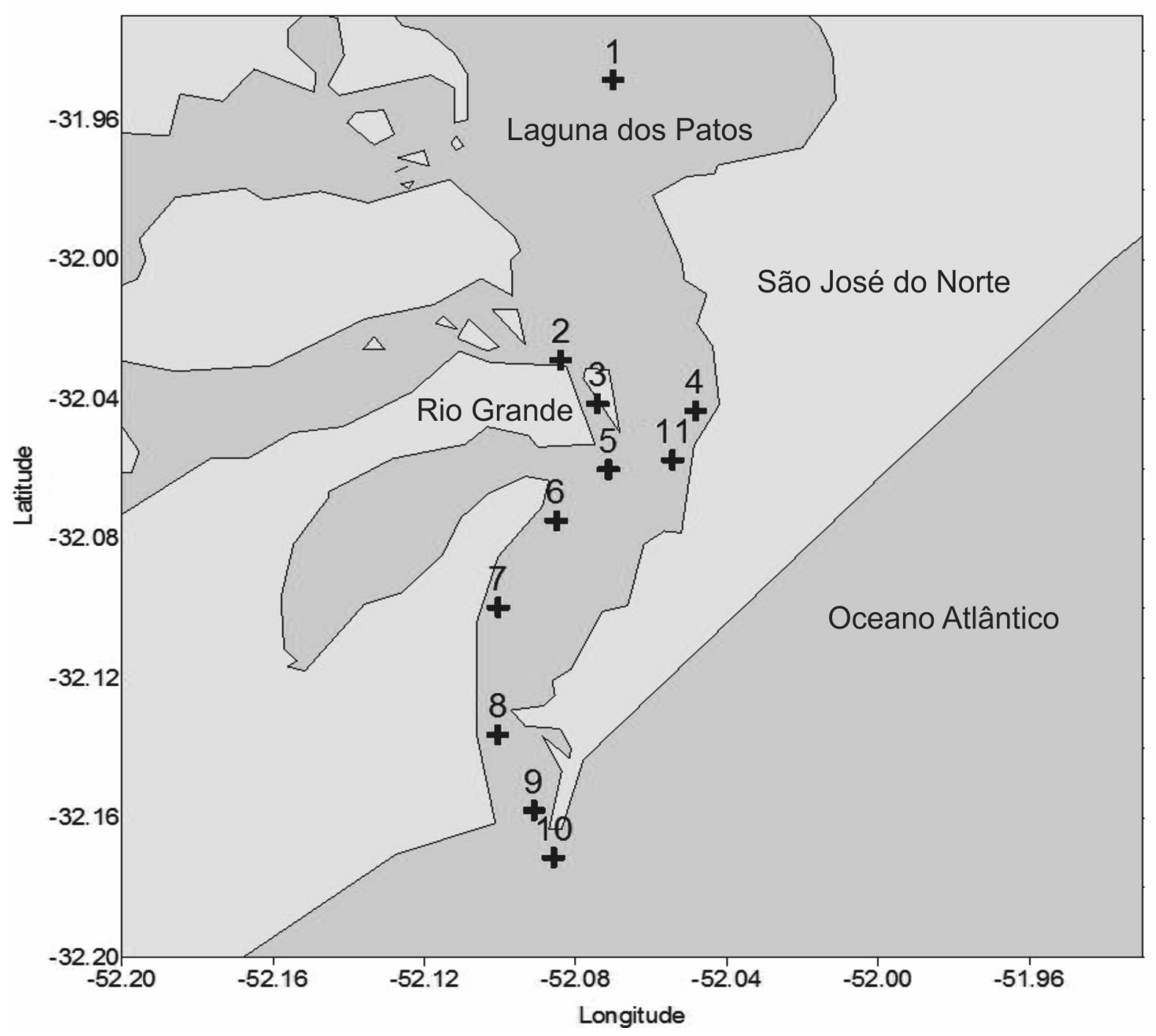

Figura 1S. Desenho amostral das 11 estações de coleta 
Tabela 1S. Concentrações de $\mathrm{As}^{3+} \mathrm{e} \mathrm{As}^{5+}\left(\mu \mathrm{g} \mathrm{L}^{-1}\right)$ na superfície da coluna d'água na Laguna dos Patos, no inverno e na primavera, seguidas de suas médias, desvios padrão, valores máximo e mínimo

\begin{tabular}{ccccc}
\hline \multirow{2}{*}{ Estações } & \multicolumn{2}{c}{ Inverno } & \multicolumn{2}{c}{ Primavera } \\
\cline { 2 - 5 } & $\mathrm{As}^{3+}\left(\mu \mathrm{g} \mathrm{L}^{-1}\right)$ & $\mathrm{As}^{5+}\left(\mu \mathrm{g} \mathrm{L}^{-1}\right)$ & $\mathrm{As}^{3+}\left(\mu \mathrm{L} \mathrm{L}^{-1}\right)$ & $\mathrm{As}^{5+}\left(\mu \mathrm{g} \mathrm{L}^{-1}\right)$ \\
\hline estação 1 & 0,27 & 2,258 & 0,55 & 1,08 \\
estação 2 & 0,243 & 2,623 & 0,612 & 1,299 \\
estação 3 & 0,226 & 2,302 & 0,579 & 1,331 \\
estação 4 & 0,253 & 2,051 & 0,557 & 0,904 \\
estação 5 & 0,214 & 1,921 & 0,58 & 0,881 \\
estação 6 & 0,25 & 1,604 & 0,583 & 0,934 \\
estação 7 & 0,222 & 2,531 & 0,545 & 1,927 \\
estação 8 & 0,212 & 2,428 & 0,543 & 2,041 \\
estação 9 & 0,218 & 1,636 & 0,536 & 1,262 \\
estação 10 & 0,249 & 2,279 & 0,59 & 1,714 \\
estação 11 & 0,22 & 2,758 & 0,583 & 1,777 \\
média & 0,234 & 2,217 & 0,569 & 1,377 \\
Desvio padrão & 0,019 & 0,380 & 0,024 & 0,423 \\
valor máximo & 0,27 & 2,758 & 0,612 & 2,041 \\
valor mínimo & 0,212 & 1,604 & 0,536 & 0,881 \\
\hline
\end{tabular}

Tabela 2S. Concentrações de $\mathrm{As}^{3+} \mathrm{e} \mathrm{As}^{5+}$ nas 11 estações de coleta ao longo da Laguna dos Patos no fundo da coluna d'água, no inverno e na primavera, seguidas de suas médias, desvios padrão, valores máximo e mínimo

\begin{tabular}{ccccc}
\hline \multirow{2}{*}{ Estações } & \multicolumn{2}{c}{ Inverno } & \multicolumn{2}{c}{ Primavera } \\
\cline { 2 - 5 } & $\mathrm{As}^{3+}\left(\mu \mathrm{g} \mathrm{L}{ }^{-1}\right)$ & $\mathrm{As}^{5+}\left(\mu \mathrm{g} \mathrm{L}^{-1}\right)$ & $\mathrm{As}^{3+}\left(\mu \mathrm{g} \mathrm{L}^{-1}\right)$ & $\mathrm{As}^{5+}\left(\mu \mathrm{g} \mathrm{L}^{-1}\right)$ \\
\hline estação 1 & 0,259 & 2,045 & 0,546 & 0,802 \\
estação 2 & 0,249 & 2,223 & 0,726 & 1,634 \\
estação 3 & 0,228 & 1,626 & 0,54 & 1,932 \\
estação 4 & 0,252 & 2,108 & 0,542 & 0,975 \\
estação 5 & 0,259 & 2,606 & 0,511 & 1,343 \\
estação 6 & 0,205 & 2,436 & 0,544 & 0,636 \\
estação 7 & 0,223 & 2,586 & 0,599 & 1,367 \\
estação 8 & 0,233 & 1,621 & 0,577 & 1,389 \\
estação 9 & 0,2 & 2,048 & 0,586 & 1,268 \\
estação 10 & 0,228 & 1,402 & 0,565 & 1,682 \\
estação 11 & 0,204 & 1,538 & 0,585 & 1,494 \\
média & 0,230 & 2,021 & 0,574 & 1,320 \\
desvio-padrão & 0,021 & 0,426 & 0,056 & 0,387 \\
valor máximo & 0,259 & 2,606 & 0,726 & 1,932 \\
valor mínimo & 0,2 & 1,402 & 0,511 & 0,636 \\
\hline
\end{tabular}

Tabela 3S. Resultados dos parâmetros químicos das amostras na superfície da coluna d'água no inverno na Laguna dos Patos

\begin{tabular}{|c|c|c|c|c|c|c|c|c|c|c|c|}
\hline Parâmetros & Unidade & Est.1 & Est.2 & Est.3 & Est.4 & Est.5 & Est.7 & Est. 8 & Est.9 & Est.10 & Est.11 \\
\hline Cond. & --- & 1,04 & 0,87 & 0,20 & 1,04 & 0,27 & 0,98 & 1,07 & 0,35 & 2,78 & 0,14 \\
\hline $\mathrm{pH}$ & --- & 7,3 & 7,3 & 7,6 & 7,2 & 7,3 & 7,1 & 7,4 & 7,4 & 7,4 & 7,9 \\
\hline Sat. $\mathrm{O}_{2}$ & $\%$ & 103,8 & 94,9 & 95,8 & 98,8 & 94,9 & 98,8 & 98,8 & 98,5 & 99,3 & 98,0 \\
\hline MS & $\mathrm{mg} \mathrm{L}^{-1}$ & 102,7 & 58,7 & 90,7 & 34,0 & 98,0 & 105,3 & 102,7 & 53,3 & 64,7 & 102,7 \\
\hline $\mathrm{NH}_{4}^{+}$ & $\mu \mathrm{mol} \mathrm{L}-1 \mathrm{em} \mathrm{N}$ & 2,70 & 3,00 & 5,00 & 2,30 & 3,70 & 3,40 & 3,07 & 2,53 & 2,27 & 3,37 \\
\hline $\mathrm{PO}_{4}^{3-}$ & $\mu \mathrm{mol} \mathrm{L}{ }^{-1} \mathrm{em} \mathrm{P}$ & 0,38 & 0,42 & 0,16 & 0,29 & 0,16 & 0,27 & 0,39 & 0,34 & 0,24 & 0,46 \\
\hline $\mathrm{P}_{\mathrm{T}}$ & $\mathrm{mg} \mathrm{L}^{-1}$ & 0,075 & 0,153 & 0,089 & 0,094 & 0,094 & 0,082 & 0,091 & 0,102 & 0,102 & 0,096 \\
\hline $\mathrm{NO}_{2}^{-}$ & $\mu \mathrm{mol} \mathrm{L} \mathrm{L}^{-1} \mathrm{em} \mathrm{N}$ & 0,33 & 0,56 & 0,55 & 0,45 & 0,47 & 0,68 & 0,43 & 0,44 & 0,38 & 0,56 \\
\hline $\mathrm{NO}_{3}^{-}$ & $\mu \mathrm{mol} \mathrm{L} \mathrm{L}^{-1} \mathrm{em} \mathrm{N}$ & 5,40 & 6,06 & 4,10 & 3,92 & 6,58 & 3,93 & 5,17 & 3,71 & 4,25 & 5,16 \\
\hline $\mathrm{SiO}_{4}{ }^{4-}$ & $\mu \mathrm{mol} \mathrm{L} \mathrm{L}^{-1} \mathrm{em} \mathrm{Si}$ & 103,7 & 89,0 & 120,8 & 91,2 & 120,6 & 88,0 & 109,5 & 73,6 & 115,0 & 106,2 \\
\hline
\end{tabular}

Tabela 4S. Resultados dos parâmetros químicos analisados no fundo da coluna d'água no inverno na Laguna dos Patos

\begin{tabular}{|c|c|c|c|c|c|c|c|c|c|c|c|}
\hline Parâmetros & Unidade & Est.1 & Est.2 & Est.3 & Est.4 & Est.5 & Est.7 & Est. 8 & Est.9 & Est.10 & Est.11 \\
\hline Cond. & --- & 0,82 & 0,87 & 0,33 & 1,00 & 0,26 & 23,30 & 2,74 & 1,28 & 0,37 & 0,15 \\
\hline $\mathrm{pH}$ & --- & 7,4 & 7,3 & 7,6 & 7,2 & 7,3 & 7,4 & 7,3 & 7,3 & 7,3 & 7,3 \\
\hline Sat. $\mathrm{O}_{2}$ & $\%$ & 99,8 & 95,0 & 95,7 & 98,2 & 95,4 & 94,1 & 97,7 & 98,2 & 99,0 & 98,0 \\
\hline MS & $\mathrm{mg} \mathrm{L}^{-1}$ & 68,0 & 70,7 & 110,0 & 58,7 & 104,7 & 112,0 & 88,0 & 74,0 & 98,0 & 124,0 \\
\hline $\mathrm{NH}_{4}^{+}$ & $\mu \mathrm{mol} \mathrm{L} \mathrm{L}^{-1} \mathrm{em} \mathrm{N}$ & 2,37 & 3,17 & 5,17 & 2,73 & 3,27 & 3,10 & 2,47 & 4,00 & 2,23 & 3,87 \\
\hline $\mathrm{PO}_{4}^{3-}$ & $\mu \mathrm{mol} \mathrm{L} \mathrm{L}^{-1} \mathrm{em} \mathrm{P}$ & 0,28 & 0,31 & 0,14 & 0,25 & 0,32 & 0,21 & 0,23 & 0,27 & 0,20 & 0,48 \\
\hline $\mathrm{P}_{\mathrm{T}}$ & $\mathrm{mg} \mathrm{L}^{-1}$ & 0,084 & 0,144 & 0,097 & 0,091 & 0,082 & 0,069 & 0,085 & 0,102 & 0,068 & 0,095 \\
\hline $\mathrm{NO}_{2}^{-}$ & $\mu \mathrm{mol} \mathrm{L} \mathrm{L}^{-1} \mathrm{em} \mathrm{N}$ & 0,45 & 0,45 & 0,53 & 0,47 & 0,69 & 0,44 & 0,47 & 0,81 & 0,52 & 0,56 \\
\hline $\mathrm{NO}_{3}^{-}$ & $\mu \mathrm{mol} \mathrm{L} \mathrm{L}^{-1} \mathrm{em} \mathrm{N}$ & 4,45 & 5,78 & 3,12 & 2,62 & 1,47 & 5,52 & 4,16 & 4,03 & 4,21 & 4,58 \\
\hline $\mathrm{SiO}_{4}{ }^{4-}$ & $\mu \mathrm{mol} \mathrm{L}{ }^{-1} \mathrm{em} \mathrm{Si}$ & 102,5 & 70,4 & 91,4 & 57,2 & 118,2 & 102,2 & 115,0 & 109,5 & 73,6 & 82,6 \\
\hline
\end{tabular}


Tabela 5S. Resultados dos parâmetros químicos da superfície da coluna d'água na primavera na Laguna dos Patos

\begin{tabular}{|c|c|c|c|c|c|c|c|c|c|c|c|}
\hline Parâmetros & Unidade & Est.1 & Est. 2 & Est.3 & Est.4 & Est. 5 & Est.7 & Est.8 & Est.9 & Est.10 & Est.11 \\
\hline Cond. & --- & 0,77 & 0,39 & 0,17 & 0,78 & 0,17 & 0,10 & 112,20 & 0,42 & 0,33 & 0,10 \\
\hline $\mathrm{pH}$ & --- & 6,4 & 6,2 & 6,1 & 6,4 & 6,1 & 5,9 & 7,3 & 6,5 & 6,7 & 6,5 \\
\hline Sat. $\mathrm{O}_{2}$ & $\%$ & 101,1 & 91,5 & 96,3 & 100,1 & 96,7 & 101,2 & 101,7 & 101,3 & 101,2 & 101,0 \\
\hline MS & $\mathrm{mg} \mathrm{L}^{-1}$ & 29,2 & 104,0 & 78,0 & 32,0 & 55,0 & 57,3 & 62,7 & 62,5 & 94,2 & 40,0 \\
\hline $\mathrm{NH}_{4}^{+}$ & $\mu \mathrm{mol} \mathrm{L} \mathrm{L}^{-1} \mathrm{em} \mathrm{N}$ & 0,27 & 5,47 & 3,61 & 2,66 & 2,66 & 1,76 & 2,35 & 1,01 & 1,60 & 1,19 \\
\hline $\mathrm{PO}_{4}^{3-}$ & $\mu \mathrm{mol} \mathrm{L} \mathrm{L}^{-1} \mathrm{em} \mathrm{P}$ & 0,76 & 1,19 & 0,48 & 0,74 & 0,56 & 0,66 & 0,66 & 0,67 & 0,67 & 0,69 \\
\hline $\mathrm{P}_{\mathrm{T}}$ & $\mathrm{mg} \mathrm{L}^{-1}$ & 0,079 & 0,073 & 0,062 & 0,076 & 0,066 & 0,064 & 0,080 & 0,066 & 0,118 & 0,069 \\
\hline $\mathrm{NO}_{2}^{-}$ & $\mu \mathrm{mol} \mathrm{L}-1$ em $\mathrm{N}$ & 0,48 & 0,61 & 0,76 & 0,28 & 0,35 & 0,36 & 0,39 & 0,30 & 0,33 & 0,31 \\
\hline $\mathrm{NO}_{3}^{-}$ & $\mu \mathrm{mol} \mathrm{L}{ }^{-1} \mathrm{em} \mathrm{N}$ & 4,36 & 5,32 & 5,10 & 4,72 & 6,05 & 5,19 & 6,05 & 4,12 & 4,40 & 5,49 \\
\hline $\mathrm{SiO}_{4}^{4-}$ & $\mu \mathrm{mol} \mathrm{L} \mathrm{L}^{-1} \mathrm{em} \mathrm{Si}$ & 103,5 & 103,4 & 63,0 & 13,9 & 82,6 & 152,5 & 105,1 & 28,5 & 37,8 & 30,5 \\
\hline
\end{tabular}

Tabela 6S. Resultados das análises dos parâmetros químicos nas amostras do fundo da coluna d'água no período da primavera

\begin{tabular}{|c|c|c|c|c|c|c|c|c|c|c|c|}
\hline Parâmetros & Unidade & Est.1 & Est. 2 & Est.3 & Est.4 & Est.5 & Est.7 & Est. 8 & Est.9 & Est.10 & Est.11 \\
\hline Cond. & --- & 0,72 & 0,41 & 0,11 & 0,81 & 0,14 & 36,00 & 10,31 & 3,46 & 25,10 & 0,10 \\
\hline $\mathrm{pH}$ & --- & 6,5 & 6,3 & 6,3 & 6,4 & 6,2 & 7,9 & 7,2 & 7,1 & 7,3 & 6,6 \\
\hline Sat. $\mathrm{O}_{2}$ & $\%$ & 101,2 & 93,0 & 97,9 & 100,4 & 97,7 & 90,9 & 98,9 & 99,6 & 95,2 & 101,1 \\
\hline MS & $\mathrm{mg} \mathrm{L}^{-1}$ & 198,5 & 123,0 & 86,2 & 269,2 & 167,5 & 220,7 & 50,5 & 58,5 & 59,2 & 15,3 \\
\hline $\mathrm{NH}_{4}^{+}$ & $\mu \mathrm{mol} \mathrm{L} \mathrm{L}^{-1} \mathrm{em} \mathrm{N}$ & 3,09 & 6,39 & 8,15 & 2,71 & 2,30 & 3,45 & 2,07 & 1,99 & 1,81 & 1,92 \\
\hline $\mathrm{PO}_{4}^{3-}$ & $\mu \mathrm{mol} \mathrm{L} \mathrm{L}^{-1} \mathrm{em} \mathrm{P}$ & 0,94 & 0,72 & 0,77 & 0,63 & 0,69 & 0,79 & 0,61 & 0,84 & 0,49 & 0,77 \\
\hline $\mathrm{P}_{\mathrm{T}}$ & $\mathrm{mg} \mathrm{L}^{-1}$ & 0,088 & 0,077 & 0,058 & 0,080 & 0,073 & 0,063 & 0,083 & 0,072 & 0,051 & 0,063 \\
\hline $\mathrm{NO}_{2}^{-}$ & $\mu \mathrm{mol} \mathrm{L} \mathrm{L}^{-1} \mathrm{em} \mathrm{N}$ & 0,36 & 0,55 & 0,33 & 0,43 & 0,54 & 0,53 & 0,35 & 0,41 & 0,34 & 0,46 \\
\hline $\mathrm{NO}_{3}^{-}$ & $\mu \mathrm{mol} \mathrm{L}-1$ em N & 5,69 & 5,27 & 3,56 & 5,28 & 4,73 & 5,37 & 4,87 & 4,59 & 4,29 & 6,00 \\
\hline $\mathrm{SiO}_{4}^{4-}$ & $\mu \mathrm{mol} \mathrm{L} \mathrm{L}^{-1} \mathrm{em} \mathrm{Si}$ & 109,5 & 105,6 & 121,5 & 11,9 & 76,7 & 155,2 & 45,2 & 31,5 & 115,4 & 102,4 \\
\hline
\end{tabular}

Tabela 7S. Autovalores e percentual da variância explicada de cada fator para o inverno (superfície da coluna d'água)

\begin{tabular}{ccccc}
\hline Fatores & Autovalores & $\begin{array}{c}\text { \% da variância } \\
\text { explicada }\end{array}$ & $\begin{array}{c}\text { Autovalores } \\
\text { acumulados }\end{array}$ & $\begin{array}{c}\text { \% da variância } \\
\text { explicada } \\
\text { acumulada }\end{array}$ \\
\hline $\mathbf{1}$ & 3,339021 & $\mathbf{2 7 , 8 2 5 1 7}$ & 3,33902 & $\mathbf{2 7 , 8 2 5 2}$ \\
$\mathbf{2}$ & 2,423031 & $\mathbf{2 0 , 1 9 1 9 3}$ & 5,76205 & $\mathbf{4 8 , 0 1 7 1}$ \\
$\mathbf{3}$ & 1,713950 & $\mathbf{1 4 , 2 8 2 9 1}$ & 7,47600 & $\mathbf{6 2 , 3 0 0 0}$ \\
$\mathbf{4}$ & 1,415345 & $\mathbf{1 1 , 7 9 4 5 5}$ & 8,89135 & $\mathbf{7 4 , 0 9 4 6}$ \\
$\mathbf{5}$ & 1,173467 & $\mathbf{9 , 7 7 8 8 9}$ & 10,06481 & $\mathbf{8 3 , 8 7 3 5}$ \\
6 & 0,987088 & 8,22574 & 11,05190 & 92,0992 \\
7 & 0,523704 & 4,36420 & 11,57561 & 96,4634 \\
8 & 0,292788 & 2,43990 & 11,86839 & 98,9033 \\
9 & 0,106116 & 0,88430 & 11,97451 & 99,7876 \\
10 & 0,025489 & 0,21241 & 12,00000 & 100,0000 \\
\hline
\end{tabular}

Tabela 9S. Autovalores e percentual de variância explicada de cada fator para a superfície da coluna d'água na primavera

\begin{tabular}{ccccc}
\hline Fatores & Autovalores & $\begin{array}{c}\text { \% da variância } \\
\text { explicada }\end{array}$ & $\begin{array}{c}\text { Autovalores } \\
\text { acumulados }\end{array}$ & $\begin{array}{c}\text { \% da variância } \\
\text { explicada } \\
\text { acumulada }\end{array}$ \\
\hline $\mathbf{1}$ & 4,030713 & $\mathbf{3 3 , 5 8 9 2 7}$ & 4,03071 & $\mathbf{3 3 , 5 8 9 3}$ \\
$\mathbf{2}$ & 2,592862 & $\mathbf{2 1 , 6 0 7 1 8}$ & 6,62357 & $\mathbf{5 5 , 1 9 6 5}$ \\
$\mathbf{3}$ & 1,916886 & $\mathbf{1 5 , 9 7 4 0 5}$ & 8,54046 & $\mathbf{7 1 , 1 7 0 5}$ \\
$\mathbf{4}$ & 1,081596 & $\mathbf{9 , 0 1 3 3 0}$ & 9,62206 & $\mathbf{8 0 , 1 8 3 8}$ \\
5 & 0,818680 & 6,82233 & 10,44074 & 87,0061 \\
6 & 0,693708 & 5,78090 & 11,13444 & 92,7870 \\
7 & 0,391374 & 3,26145 & 11,52582 & 96,0485 \\
8 & 0,308561 & 2,57134 & 11,83438 & 98,6198 \\
9 & 0,114403 & 0,95336 & 11,94878 & 99,5732 \\
10 & 0,051217 & 0,42681 & 12,00000 & 100,0000 \\
\hline
\end{tabular}

Tabela 8S. Autovalores e percentual da variância explicada de cada fator para o inverno (fundo da coluna d'água)

\begin{tabular}{ccccc}
\hline Fatores & Autovalores & $\begin{array}{c}\text { \% da variância } \\
\text { explicada }\end{array}$ & $\begin{array}{c}\text { Autovalores } \\
\text { acumulados }\end{array}$ & $\begin{array}{c}\text { \% da variância } \\
\text { explicada } \\
\text { acumulada }\end{array}$ \\
\hline $\mathbf{1}$ & 2,683949 & $\mathbf{2 2 , 3 6 6 2 4}$ & 2,68395 & $\mathbf{2 2 , 3 6 6 2}$ \\
$\mathbf{2}$ & 2,305388 & $\mathbf{1 9 , 2 1 1 5 7}$ & 4,98934 & $\mathbf{4 1 , 5 7 7 8}$ \\
$\mathbf{3}$ & 1,968087 & $\mathbf{1 6 , 4 0 0 7 3}$ & 6,95742 & $\mathbf{5 7 , 9 7 8 5}$ \\
$\mathbf{4}$ & 1,692218 & $\mathbf{1 4 , 1 0 1 8 2}$ & 8,64964 & $\mathbf{7 2 , 0 8 0 4}$ \\
$\mathbf{5}$ & 1,582638 & $\mathbf{1 3 , 1 8 8 6 5}$ & 10,23228 & $\mathbf{8 5 , 2 6 9 0}$ \\
6 & 0,773777 & 6,44814 & 11,00606 & 91,7171 \\
7 & 0,590093 & 4,91744 & 11,59615 & 96,6346 \\
8 & 0,263364 & 2,19470 & 11,85952 & 98,8293 \\
9 & 0,111359 & 0,92799 & 11,97087 & 99,7573 \\
10 & 0,029126 & 0,24272 & 12,00000 & 100,0000 \\
\hline
\end{tabular}

Tabela 10S. Autovalores e percentual da variância explicada de cada fator para o fundo da coluna d'água na primavera

\begin{tabular}{ccccc}
\hline Fatores & Autovalores & $\begin{array}{c}\text { \% da variância } \\
\text { explicada }\end{array}$ & $\begin{array}{c}\text { Autovalores } \\
\text { acumulados }\end{array}$ & $\begin{array}{c}\text { \% da variância } \\
\text { explicada } \\
\text { acumulada }\end{array}$ \\
\hline $\mathbf{1}$ & 4,234064 & $\mathbf{3 5 , 2 8 3 8 6}$ & 4,23406 & $\mathbf{3 5 , 2 8 3 9}$ \\
$\mathbf{2}$ & 2,190969 & $\mathbf{1 8 , 2 5 8 0 8}$ & 6,42503 & $\mathbf{5 3 , 5 4 1 9}$ \\
$\mathbf{3}$ & 2,037055 & $\mathbf{1 6 , 9 7 5 4 6}$ & 8,46209 & $\mathbf{7 0 , 5 1 7 4}$ \\
$\mathbf{4}$ & 1,111318 & $\mathbf{9 , 2 6 0 9 8}$ & 9,57341 & $\mathbf{7 9 , 7 7 8 4}$ \\
5 & 0,997788 & 8,31490 & 10,57119 & 88,0933 \\
6 & 0,716979 & 5,97482 & 11,28817 & 94,0681 \\
7 & 0,428331 & 3,56943 & 11,71650 & 97,6375 \\
8 & 0,175197 & 1,45997 & 11,89170 & 99,0975 \\
9 & 0,071063 & 0,59219 & 11,96276 & 99,6897 \\
10 & 0,037236 & 0,31030 & 12,00000 & 100,0000 \\
\hline
\end{tabular}

